\title{
High-grade carotid artery stenosis: A forgotten area in cardiovascular risk management
}

Elin Good, Toste Länne, Elisabeth Wilhelm, Joep Perk, Tiny Jaarsma and Ebo de Muinck

\author{
Journal Article
}

\section{Tweet}

N.B.: When citing this work, cite the original article.

Original Publication:

Elin Good, Toste Länne, Elisabeth Wilhelm, Joep Perk, Tiny Jaarsma and Ebo de Muinck, High-grade carotid artery stenosis: A forgotten area in cardiovascular risk management, European Journal of Preventive Cardiology, 2016. 23(13), pp.1453-1460.

http://dx.doi.org/10.1177/2047487316632629

Copyright: SAGE Publications (UK and US)

http://www.uk.sagepub.com/home.nav

Postprint available at: Linköping University Electronic Press

http://urn.kb.se/resolve?urn=urn:nbn:se:liu:diva-129961

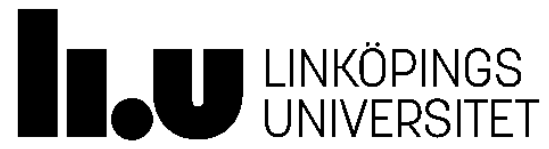




\title{
High-grade carotid artery stenosis: a forgotten area in cardiovascular risk factor management
}

\author{
Elin Good ${ }^{1}$, Toste Länne ${ }^{1}$, Elisabeth Wilhelm ${ }^{1}$, Joep Perk ${ }^{2}$, Tiny Jaarsma ${ }^{3}$ \\ and Ebo de Muinck ${ }^{1}$
}

${ }^{1}$ Department of Medicine and Health Sciences, Linköping University, Sweden

${ }^{2}$ Department of Health and Caring Sciences, Linnaeus University, Sweden

${ }^{3}$ Department of Social and Welfare Studies, Linköping University, Sweden

\section{Corresponding Author:}

Elin Good, Department of Medicine and Health Sciences, Division of Cardiovascular Medicine

Linköping University

SE-581 85 Linköping

Sweden.

Email: elin.good@liu.se 


\begin{abstract}
Background: Patients with high-grade $(\geq 70 \%)$ carotid artery stenosis (CAS) rank in the highest risk category for future cardiovascular (CV) events, but the quality of cardiovascular risk management in this group is unknown.
\end{abstract}

Design: Cross-sectional retrospective study.

Methods: In all patients with high-grade CAS diagnosed in Östergötland county, Sweden between January $1^{\text {st }}, 2009$ and June 31 ${ }^{\text {st }}$, 2012, data were collected regarding quality of cardiovascular risk management, co-morbidity and outcomes during 2-year follow-up after carotid ultrasound diagnosis of CAS. Patients were included regardless of whether they underwent carotid endarterectomy (CEA).

Results: 393 CAS patients were included in the study, 133 (33.8\%) underwent CEA and 260 (66.2\%) were assigned to conservative management (CM). In both groups prescription of platelet inhibitors, statins and anti-hypertensives increased significantly $(p<0.001)$ after diagnosis, however treatment targets were not met in the majority of patients and LDL level was on target in only 13.5\%. During follow-up LDL was not measured in $19.8 \%$ of CEA and $44.2 \%$ of CM patients ( $p<0.001)$, HbA1c was not measured in $24.4 \%$ of diabetics (CEA) versus $18.8 \%(\mathrm{CM})(p=0.560)$. There was no 
documentation of counseling on diet, exercise, smoking cessation and adherence to medication. The combined clinical event rate (all-cause mortality, CV mortality and non-fatal CV events) was high in both groups (CEA: 36.8\% versus CM: 36.9\%, $p=$ 1.00) with no difference in ipsilateral ischemic stroke.

Conclusions: In patients with high-grade CAS the clinical event rate is high and cardiovascular risk management is deficient in all aspects.

\section{Keywords}

high-grade carotid stenosis, cardiovascular risk management, cardiovascular disease, secondary prevention, atherosclerosis 


\section{Introduction}

According to current European guidelines on cardiovascular disease (CVD) prevention patients with high-grade ( $\geq 70 \%$ ) carotid artery stenosis (CAS) rank in the "very high risk" category for future cardiovascular (CV) events ${ }^{1}$. Prevention in high-grade CAS is focused on a single outcome, the reduction of (recurrent) ischemic stroke with an emphasis on carotid endarterectomy (CEA) surgery or stenting, antiplatelet therapy, lipid management and the treatment of hypertension ${ }^{2}$. However, within current guidelines, the concept is well established that CVRM in very high risk patients should take a multi-facetted approach and combine pharmacological management of risk factors with lifestyle modification ${ }^{1,3}$. The quality of CVRM in patients with high-grade CAS is unknown and there is no information regarding their total CVD burden. We aimed to assess the quality of CVRM in patients with high-grade CAS under routine clinical care against the background of total CV risk and clinically manifest CVD at baseline together with clinical events during two-year follow-up. We studied all patients with high-grade CAS, since all are in need of CVRM regardless of whether they undergo CEA or not. 


\section{Methods}

The study was performed at the University Hospital of Linköping, in Östergötland county, Sweden, which has a population of 445 029. Since 2009 this county has universal implementation of a single type of electronic patient record (Cambio Cosmic, Cambio Healthcare Systems, Linköping, Sweden). Thus, all medical information on every patient is available at every point of care throughout the county. This includes all consultations and hospitalizations throughout the healthcare system, all diagnostic procedures, all therapeutic interventions and every medication that has been prescribed during the patient's medical history. Taking advantage of this comprehensive medical documentation, we performed an observational, retrospective study of all patients who were diagnosed with high-grade CAS between January 1 $1^{\text {st }}, 2009$ and July 31 ${ }^{\text {st }}, 2012$. The study was approved by Linköping’s Institutional Review Board (record number 2013/463-31).

\section{Patients}

All patients with high-grade CAS were considered for inclusion in the study regardless of whether they had undergone CEA. The decision to perform CEA was taken by the vascular surgeon, based on the severity of the CAS and overall clinical condition of the patient. Patients were excluded if they had non-atherosclerotic carotid artery disease and/or were not available for follow-up because they resided outside Östergötland. 
All duplex ultrasound investigations that were performed between January $1^{\text {st }}$, 2009 and July 31 ${ }^{\text {st }}, 2012$ were screened for patients with high-grade CAS. Stenosis severity was assessed by measuring peak systolic velocity (PSV) according to criteria established in the European Carotid Surgery Trial ${ }^{4}$. Using these criteria, a PSV of $\geq 2.4$ $\mathrm{m} / \mathrm{s}$ measured at an insonation angle $\leq 60^{\circ}$ corresponds to $\mathrm{a} \geq 70 \%$ stenosis and a PSV of $0.0 \mathrm{~m} / \mathrm{s}$ indicates occlusion. Follow-up-time was two years for all patients, starting on the day that high-grade CAS was diagnosed.

\section{Study aims and measurements}

The primary aim of the study was to assess the number of patients who fulfilled treatment goals for secondary prevention at baseline, i.e. before the diagnosis of highgrade CAS and during the first year after this diagnosis. The quality of CVRM was assessed in terms of prescribed medication, blood pressure, lipid and HbA1c levels, as well as documentation of counseling regarding smoking cessation, diet, and exercise. Treatment goals were set according to European Guidelines as published during the study period with blood pressure $\leq 140 / 90 \mathrm{mmHg}$, total cholesterol $\leq 5.0 \mathrm{mmol} / \mathrm{L}, \mathrm{LDL}$ cholesterol $\leq 1.8 \mathrm{mmol} / \mathrm{L}$, and $\mathrm{HbA} 1 \mathrm{c}<53 \mathrm{mmol} / \mathrm{mol}$ in diabetics ${ }^{1}$.

The secondary aim of the study was to place the quality of CVRM within the context of overall CV risk and clinically manifest CVD at baseline as well as clinical events during follow-up. To generate a CV risk-profile, we assessed for family history, 
lipid values, smoking, overconsumption of alcohol, overweight, hypertension, diabetes mellitus, atrial fibrillation and renal failure. A BMI $\geq 25$ was considered to indicate overweight and alcohol overconsumption was defined as consumption $>20 \mathrm{~g} / \mathrm{day}$ ethanol for men and $>10 \mathrm{~g} /$ day ethanol for women ${ }^{1}$. Renal failure was defined as stage $\geq 4$, i.e. glomerular filtration rate $(\mathrm{GFR})<30 \mathrm{~mL} / \mathrm{min} / 1.73 \mathrm{~m}^{2}{ }^{5}$. To assess total CVD burden we recorded clinically manifest ischemic heart disease (IHD) and lower extremity peripheral artery disease (PAD) at baseline. During two years after the diagnosis of high-grade CAS we recorded all-cause mortality, CV mortality and all nonfatal CV events.

Clinical characteristics including contra-indications to CEA, laboratory values, events and medication were recorded for each patient on dedicated case record forms. Definitions and classification of clinical events were established prior to the study (Suppl. Table 1). Treatment targets for CVD prevention were assessed during $\leq 1$ year before and $\leq 1$ year after diagnosis of high-grade CAS.

\section{Statistical analysis}

A database was created using SPSS Statistics 22 (International Business Machines Corporation, New York, NY, USA). Continuous variables were summarized as mean \pm standard deviation (SD) for values with a normal distribution and as median or interquartile range (IQR) if the values were not normally distributed. 
Differences between patients with and without CEA were tested with $\mathrm{X}^{2}$ (categorical variables), unpaired $t$ test (continuous normally distributed variables) or Mann-Whitney $U$ test (continuous skewed variables). A paired samples $t$ test was used to compare laboratory values and blood pressure levels before and after diagnosis of CAS.

All variables in the database (Suppl. Table 2) representing co-morbidities and risk factors were tested for correlation (Spearman bivariate correlation) to the combined endpoint of CV death and non-fatal CV events. A Cox proportional hazard analysis was then performed with 95\% confidence intervals (CIs) for all variables with a significant correlation, and for age and gender. Age and gender were treated as categorical variables (age: $>75$ years, or $\leq 75$ years). If a patient had multiple events, the first was used in the analysis. Patients who died from non-CV causes as first event were excluded from the analysis to avoid competing endpoints.

\section{Results}

Baseline characteristics

Between January $1^{\text {st }}, 2009$ and June $31^{\text {st }}, 2012$ a total of 7172 duplex ultrasound investigations were performed. These yielded 410 patients with high-grade CAS, 10 patients were excluded because of non-atherosclerotic carotid artery disease and seven patients resided outside Östergötland. Thus, 393 patients were included in the study. 
Table 1 summarizes the baseline characteristics, depicting an elderly population where the majority were men. For men the mean age was $73.7 \pm 9.1$ years and for women it was $74.0 \pm 9.7$ years $(p<0.01)$. In the majority, ultrasound was performed because of a cerebrovascular ischemic event (CVI) while a minority was referred for ultrasound because of a carotid bruit. Overall, the incidence of concomitant IHD was high with a significantly higher rate of IHD in those assigned to conservative management (CM). Regarding CEA, surgery was performed at a median of 4 (IQR 11) days after ultrasound. The contraindications for CEA surgery in the CM group are summarized in Supplementary Figure 1. Most prevalent were near-occlusion or occlusion of the artery (33.2\%) and asymptomatic stenosis (50.0\%).

Quality of cardiovascular risk management The risk factors at the time of CAS diagnosis, and the extent to which relevant data for risk assessment and treatment planning were not obtained are shown in Supplemental Table 3. For $12.7 \%$ there was no documentation of smoking habits. The presence or absence of alcohol overconsumption was not recorded for $55.7 \%$, the presence or absence of a BMI $\geq 25$ was not documented for $36.4 \%$ of patients and information on family history was absent.

Table 2 summarizes the changes in prescriptions of medication, lipid levels, blood pressure and HbA1c levels before and after the diagnosis of high-grade CAS as well as 
the number of patients in whom lipids, blood pressure and HbA1c were not measured either before, after or both. There were modest, but significant reductions in cholesterol, blood pressure and HbA1c levels after diagnosis of CAS. However, there was a substantial number of patients in whom these risk factors were not measured, both before and after diagnosis.

Comparison of the CEA and CM groups showed that during the first year of follow-up LDL was not measured in $19.8 \%$ of CEA and $44.2 \%$ of CM patients ( $p<$ 0.001), HbA1c was not measured in $24.4 \%$ of diabetics (CEA) versus $18.8 \%(\mathrm{CM})(p=$ 0.560). The CM group had significantly lower blood pressure (systolic pressure CM 139 $\pm 19 \mathrm{~mm} \mathrm{Hg}$ vs. CEA $144 \pm 15 \mathrm{~mm} \mathrm{Hg}, p<0.05$ and diastolic pressure CM $74 \pm 9 \mathrm{~mm}$ Hg vs. CEA $77 \pm 9$ mm Hg, $p<0.05)$. There were no significant differences between the groups regarding lipid levels and HbA1c values after diagnosis. In both groups prescription of platelet inhibitors, anti-coagulants, anti-hypertensives and lipid lowering medications increased significantly. There was no documentation of adherence to medication or life style counseling.

Figure 1 summarizes the extent to which lipid and blood pressure treatment targets were met as well as the change in prescription of lipid lowering, anti-platelet and anti-coagulant medication. Patients for whom lipid- or blood pressure values were not measured are classed as “not reaching target”. Despite a significant increase in 
prescriptions for statins, there was only a non-significant $2.8 \%$ increase in patients in whom LDL level was on target after diagnosis of CAS from $10.7 \%$ to $13.5 \%$.

Mortality and non-fatal cardiovascular events during follow-up

All-cause and CV mortality as well as non-fatal CV events are summarized in Figure 2. The over-all event rate within two years was high in both groups (CEA: 36.8\% versus CM: $36.9 \%, p=1.00)$.

All-cause mortality, mortality from stroke and non-CV mortality were significantly higher in the CM group, but there was no difference in CV mortality that was not due to stroke (Fig. 2 A-D). Cancer and infections were the major non-cardiovascular causes of death (Suppl. Table 4). There were no differences between the groups regarding non-fatal CV events including recurrent ipsilateral stroke (Fig. 2E).

Of those who experienced any CV event, the majority (68.3\%) had one event, 23.4\% had two, $4.8 \%$ had three and $3.4 \%$ had four events. In the CEA group, eight patients (6\% of CEA) had a complication directly related to CEA, most often infection in the operation wound or neurological damage, these were not included in the outcomeanalysis summarized above.

The results of the Cox regression analysis are shown in Table 3. In multivariate analysis a symptomatic CAS and the presence of heart failure significantly increased the risk for the combined endpoint of CV death and non-fatal CV events. 


\section{Discussion}

To the best of our knowledge this is the first analysis of the quality of CVRM in patients with high-grade CAS. Despite the extremely high CVD burden and the high clinical event rate during follow-up every aspect of CVRM was deficient. Furthermore, the discrepancy between prescriptions of statins and the absence of significant improvements in target cholesterol levels suggests issues with dosing/titration of medication and/or problems with adherence to pharmacotherapy.

The current data fit a pattern of suboptimal CVRM in patients with non-coronary CVD. For cerebral ischemia and PAD, CVRM is suboptimal across countries and regions ${ }^{6-9}$ and it is implemented less frequently than for CAD ${ }^{10,11}$. There are no previous studies on CVRM in CAS but in view of the systematic under-treatment of patients with other forms of non-coronary CVD, it is likely that our findings are not unique.

The literature on secondary prevention in CAS places a strong emphasis on elaborating the indications for CEA and its efficacy in preventing stroke ${ }^{2}$. Recommendations emphasize the need for "optimal medical therapy“ as an adjunct to any carotid revascularization, which should include antiplatelet therapy, statins and life style modification². However, in the current study "optimal medical therapy“ was not realized. Moreover, it is unknown to what extent CVRM in patients with high-grade CAS can reduce CV events in other vascular beds. 
Regarding the prevention of stroke, statins have shown efficacy in patients with CAS. The SPARCL trial, showed a 33\% reduction in stroke risk for those with CAS and no heterogeneity in treatment effect between those with and without CAS ${ }^{12}$. However, we show that in routine care, lipid treatment targets are met only in a minority of patients. This finding is also true for other high risk patients. A previous report on individuals with a PROCAM score $>20 \%$, CVD or diabetes, showed that only $37 \%$ met lipid targets ${ }^{13}$.

The impact of lifestyle adaptations in patients with CAS is unknown. However, the literature on PAD and cerebral ischemia may apply to some extent to CAS. Smoking cessation has been shown to increase survival twofold in PAD ${ }^{14}$. However, the effect may be heterogeneous by vascular bed and therefore difficult to extrapolate to CAS ${ }^{14}$. For alcohol there is a J-shaped association between intake and CVD risk including ischemic stroke. Moderate intake (1 drink/day for women, 2 drinks/day for men) is protective but heavier use increases risk ${ }^{1,2}$. Thus in CAS heavy drinking should be discouraged and light to moderate drinking may be reasonable. A Mediterranean diet is associated with reduced CV and all-cause mortality ${ }^{1}$. Regarding secondary prevention of stroke however, there are no data on the effectiveness of specific diets ${ }^{2}$. Thus the extent to which a Mediterranean diet offers protection in CAS especially regarding stroke remains to be established. Regular physical activity reduces CV 
mortality, and may reduce stroke risk ${ }^{1,2}$. Therefore, disability from stroke should not deter from establishing a suitable exercise regimen in patients with CAS.

The best manner in which to optimize CVRM for patients with high grade CAS is not known. However, structured CVRM programs such as offered to patients after myocardial infarction have been shown to be particularly important and cost-effective ${ }^{1}$. The sustained contact offered in these programs has been proven effective in facilitating dose optimization of medication and promoting adherence ${ }^{1}$. This may be especially beneficial for the patients documented here because of the discrepancy between statin prescriptions and attainment of cholesterol targets.

The current study shows no difference in the recurrence of ipsilateral CVI between the CEA and CM group. However, the groups may not have been comparable regarding stroke risk. In the CM group $72 \%$ of patients had an asymptomatic CAS, which is associated with a lower stroke risk than symptomatic stenosis ${ }^{15}$. On the other hand patients in the CM group had significantly more IHD which increases stroke risk threefold ${ }^{16}$. CEA has a role in secondary prevention after a CVI but without effective CVRM the beneficial effect is likely to be only be partially realized.

We acknowledge the limitations of the current study. The retrospective nature of the analysis constrained the assessment of a number of variables. Family history and adherence to medication were not recorded, we found gaps in the documentation of risk factors related to life style and there was no record showing that life style changes were 
implemented. This does not necessarily mean that life style and adherence were not addressed, but data on these parameters might have been collected more successfully in a prospective study. The medical record is complete on all other aspects of care. We would argue therefore, that the current data represent a clinically relevant assessment of the quality of CVRM in patients with high grade CAS.

\section{Conclusion}

- Cardiovascular risk management in patients with high-grade CAS is deficient in all aspects. This is illustrated by a wide gap in prescription of preventive medication and the attainment of treatment targets.

- Improved cardiovascular risk management in this group is mandated by the extremely high CVD risk as reflected both by the high CVD burden at baseline and a high incidence of clinical manifestations of polyvascular disease at baseline as well as during follow-up.

- In view of the deficient cardiovascular risk management in this group, the clinical benefits of carotid endarterectomy might improve once it is accompanied by optimal cardiovascular risk management. 


\section{Declaration of conflicting interests}

None declared.

\section{Funding acknowledgements}

This work was supported by Linköping University Hospital [grant number: LIO417951]. 


\section{References}

1. Perk J, De Backer G, Gohlke H, et al. European Guidelines on cardiovascular disease prevention in clinical practice (version 2012). The Fifth Joint Task Force of the European Society of Cardiology and Other Societies on Cardiovascular Disease Prevention in Clinical Practice (constituted by representatives of nine societies and by invited experts). Eur Heart J. 2012; 33: 1635-701.

2. Kernan WN, Ovbiagele B, Black HR, et al. Guidelines for the prevention of stroke in patients with stroke and transient ischemic attack: a guideline for healthcare professionals from the American Heart Association/American Stroke Association. Stroke. 2014; 45: 2160-236.

3. Piepoli MF, Corra U, Adamopoulos S, et al. Secondary prevention in the clinical management of patients with cardiovascular diseases. Core components, standards and outcome measures for referral and delivery: a policy statement from the cardiac rehabilitation section of the European Association for Cardiovascular Prevention \& Rehabilitation. Endorsed by the Committee for Practice Guidelines of the European Society of Cardiology. Eur J Prev Cardiol. 2014; 21: 664-81.

4. Randomised trial of endarterectomy for recently symptomatic carotid stenosis: final results of the MRC European Carotid Surgery Trial (ECST). Lancet. 1998; 351: 1379-87.

5. Schiffrin EL, Lipman ML and Mann JF. Chronic kidney disease: effects on the cardiovascular system. Circulation. 2007; 116: 85-97.

6. Asberg S, Henriksson KM, Farahmand B, et al. Ischemic stroke and secondary prevention in clinical practice: a cohort study of 14,529 patients in the Swedish Stroke Register. Stroke. 2010; 41: 133842.

7. Ramsay SE, Whincup PH, Wannamethee SG, et al. Missed opportunities for secondary prevention of cerebrovascular disease in elderly British men from 1999 to 2005: a population-based study. J Public Health (Oxf). 2007; 29: 251-7. 
8. Al Banna M, Baldawi H, Kadhim A, Humaidan H and Whitford DL. Stroke in Bahrain: rising incidence, multiple risk factors, and suboptimal care. Int J Stroke. 2015; 10: 615-8.

9. Flu HC, Tamsma JT, Lindeman JH, Hamming JF and Lardenoye JH. A systematic review of implementation of established recommended secondary prevention measures in patients with PAOD. Eur J Vasc Endovasc Surg. 2010; 39: 70-86.

10. Bejot Y, Zeller M, Lorgis L, et al. Secondary prevention in patients with vascular disease. A population based study on the underuse of recommended medications. J Neurol Neurosurg Psychiatry. 2013; 84: 348-53.

11. Subherwal S, Patel MR, Kober L, et al. Missed opportunities: despite improvement in use of cardioprotective medications among patients with lower-extremity peripheral artery disease, underuse remains. Circulation. 2012; 126: 1345-54.

12. Sillesen H, Amarenco P, Hennerici MG, et al. Atorvastatin reduces the risk of cardiovascular events in patients with carotid atherosclerosis: a secondary analysis of the Stroke Prevention by Aggressive Reduction in Cholesterol Levels (SPARCL) trial. Stroke. 2008; 39: 3297-302.

13. Jaussi A, Noll G, Meier B and Darioli R. Current cardiovascular risk management patterns with special focus on lipid lowering in daily practice in Switzerland. Eur J Cardiovasc Prev Rehabil. 2010; 17: 363-72.

14. Bonaca MP and Creager MA. Pharmacological treatment and current management of peripheral artery disease. Circ Res. 2015; 116: 1579-98.

15. European Stroke O, Tendera M, Aboyans V, et al. ESC Guidelines on the diagnosis and treatment of peripheral artery diseases: Document covering atherosclerotic disease of extracranial carotid and vertebral, mesenteric, renal, upper and lower extremity arteries: the Task Force on the Diagnosis and Treatment of Peripheral Artery Diseases of the European Society of Cardiology (ESC). Eur Heart J. 2011; 32: 2851-906. 
16. Kannel WB, Wolf PA and Verter J. Manifestations of coronary disease predisposing to stroke. The Framingham study. JAMA. 1983; 250: 2942-6. 
Figure 1. Prescription of medication and treatment targets before and after diagnosis of high-grade carotid artery stenosis.

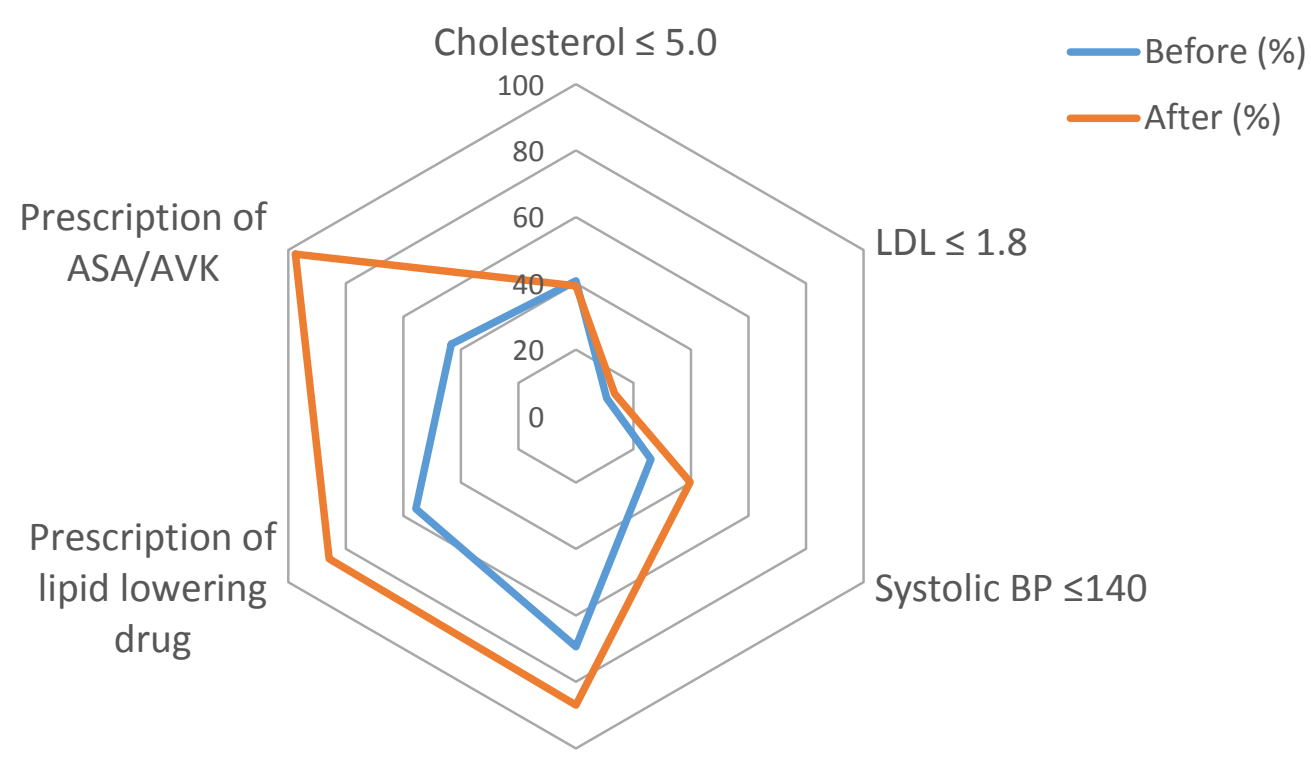

Diastolic BP $\leq 90$

ASA, Acetyl salicylic acid; AVK, Anti-vitamin K drug; LDL, Low-density lipoprotein; BP, Blood pressure; CAS, Carotid artery stenosis.

Percentages of patients who reached target goals $\leq$ one year before- and $\geq$ one year after diagnosis of highgrade CAS. Patients without registered values are considered as not having met treatment targets. Pre-post differences were significant for prescription of ASA/AVK $(p<0.001)$ and lipid lowering drugs $(p<0.001)$, 
systolic blood pressure on target $(p<0.005)$ and diastolic blood pressure on target $(p<0.05)$, but not for the number of patients on target for LDL $(p=0.286)$ and cholesterol $(p=0.775)$. 
Table 1. Baseline characteristics.

\begin{tabular}{lccc}
\hline & CEA & CM & $p$ \\
\hline Gender, N (\%) & & & \\
Male & & & \\
\multicolumn{2}{l}{ M $=260$} & & \\
\end{tabular}

Age

Age, mean (SD)

$72.0(9.5) \quad 74.7(9.1) \quad<0.01$

Location of plaque, N (\%)

$\begin{array}{llll}\text { Right } & 58(43.6) & 124(47.7) & 0.640 \\ \text { Left } & 53(39.8) & 108(41.5) & 0.835 \\ \text { Bilateral } & 22(16.5) & 28(10.8) & 0.156\end{array}$

Symptomatic Stenosis, N (\%)

Yes

$114(85.7) \quad 73(28.1) \quad<0.001$

Severity of Stenosis, n (\%)

70-89 \% stenosis

90-99\% stenosis

Occlusion

Data not available

CT scan of the brain, $\mathrm{N}(\%)$
At time of diagnosis
214 (82.3)
0.797
With signs of chronic ischemia
30 (26.3)
$69(32.2)$
0.411
With signs of previous silent infarction
35 (30.7)
$67(31.3) \quad 1.000$ 
Reason for carotid ultrasound, N (\%)

\begin{tabular}{|c|c|c|c|}
\hline CVI & $92(69.2)$ & $156(60.0)$ & 0.400 \\
\hline Suspicion of CVI & $16(12.0)$ & $24(9.2)$ & 0.435 \\
\hline $\begin{array}{l}\text { Non-specific/non-focal neurologic } \\
\text { symptoms }\end{array}$ & $6(4.5)$ & $21(8.1)$ & 0.215 \\
\hline Carotid bruit & $5(3.8)$ & $17(6.5)$ & 0.282 \\
\hline Other reasons & $14(10.5)$ & $42(16.2)$ & 0.187 \\
\hline \multicolumn{4}{|l|}{ morbidities, N (\%) } \\
\hline Previous CVI & $121(91.0)$ & $209(80.4)$ & 0.428 \\
\hline Ischemic heart disease & $41(30.8)$ & 135 (51.9) & $<0.05$ \\
\hline Peripheral vascular disease & $20(15.0)$ & $52(20.0)$ & 0.314 \\
\hline Diabetes mellitus & $41(30.8)$ & 85 (32.7) & 0.787 \\
\hline Renal failure & $5(3.8)$ & $19(7.3)$ & 0.189 \\
\hline Atrial fibrillation & $23(17.3)$ & $63(24.2)$ & 0.203 \\
\hline
\end{tabular}

CEA, Carotid Endarterectomy; CM, Conservative Management, N, Number of patients; n, number of plaques; CT, Computer Tomography; CVI, Cerebrovascular Ischemic event.

Baseline characteristics of the patients at time of diagnosis of high-grade carotid stenosis divided in two groups: those who underwent carotid endarterectomy versus those who received conservative management. Data for CT scans with chronic ischemia and previous silent infarction are expressed as percentage of all patients who underwent a CT scan at the time of diagnosis, not as a percentage of the population as a whole. 
Table 2. Lipid levels, blood pressure, glycaemic control and medication before and after diagnosis of high-grade carotid artery stenosis

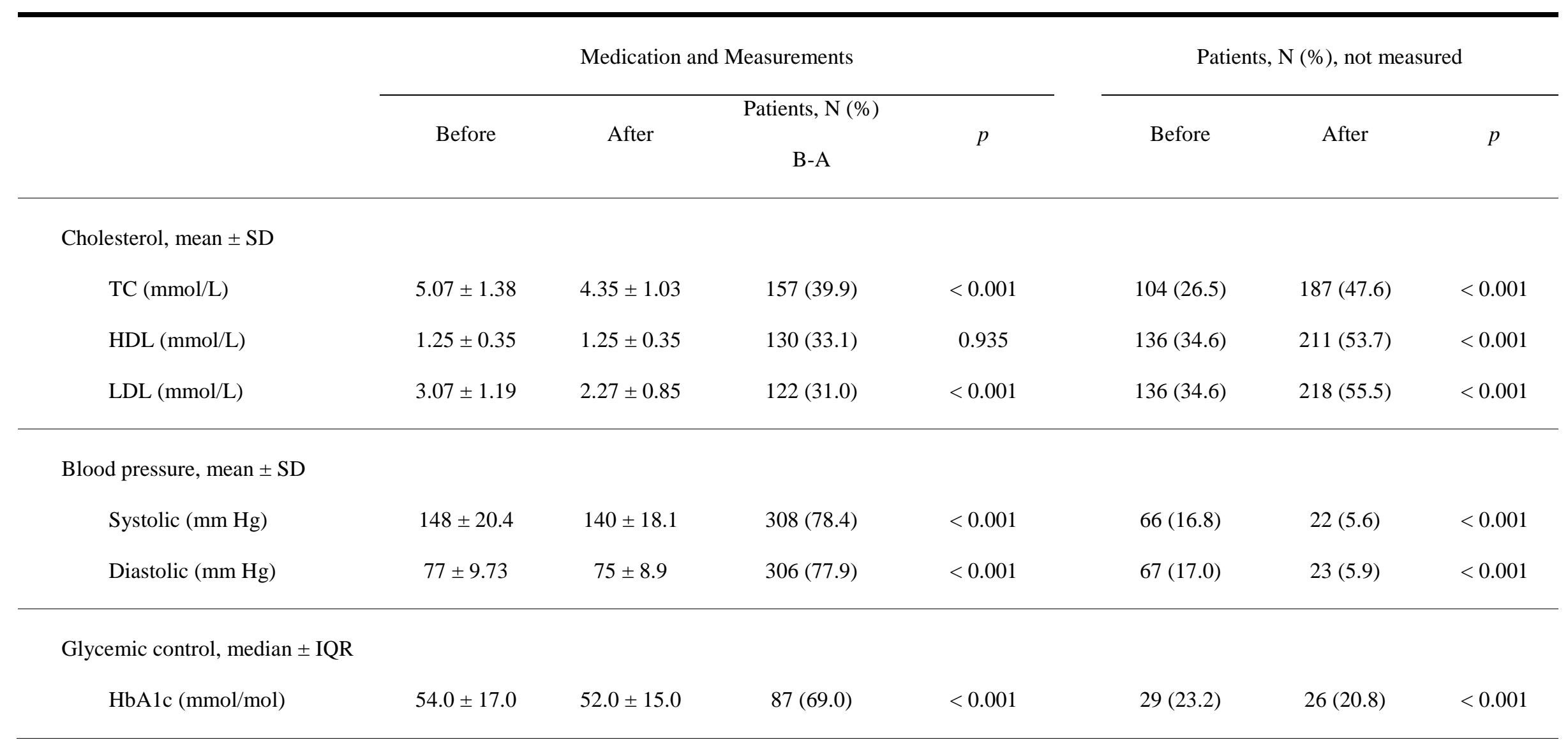


Prescriptions, N (\%)

$\begin{array}{lcccc}\text { Any platelet inhibitor } & 229(58.3) & 329(83.7) & \text { n.a. } & <0.001 \\ \text { Any anticoagulant } & 43(10.9) & 67(17.0) & \text { n.a. } & <0.001 \\ \text { Any antihypertensive } & 294(74.8) & 342(87.0) & \text { n.a. } & <0.001 \\ \text { Any lipid lowering med. } & 219(55.7) & 337(85.8) & \text { n.a. } & <0.001\end{array}$

N, Number of patients; B-A, Number of patients who had at least one measurement before and after diagnosis; SD, Standard deviation; TC, Total cholesterol;

HDL, High-density lipoprotein; LDL, Low-density lipoprotein; IQR, Inter quartile range; med, medication; n.a., Not applicable.

Risk factor levels and prescriptions in those with at least one measurement $\leq 1$ year before the diagnosis of high-grade carotid artery stenosis and one measurement $\leq 1$ year after diagnosis. Also shown is the number of patients in whom these measurements were not performed during these time frames. HbA1clevels are measured only for patients with diabetes mellitus. A diagnosis of diabetes was present in 126 patients (32.1\%). Before-after measurements not applicable to prescriptions because of the nominal nature of these data, patients either had a prescription or not. 
Table 3. Univariate and multivariate Cox regression analysis for the combined endpoint of cardiovascular death and nonfatal cardiovascular events.

\begin{tabular}{|c|c|c|c|c|}
\hline Variables & Univariate analysis RR (95\% CI) & $p$ & Multivariate analysis RR (95\% CI) & $p$ \\
\hline Age $>75$ & $1,129(0,795-1,604)$ & 0,498 & $1,023(0,704-1,488)$ & 0,905 \\
\hline Male & $0,76(0,678-1,407)$ & 0,898 & $0,931(0,643-1,350)$ & 0,707 \\
\hline Carotid Endarterectomy & $1,127(0,784-1,621)$ & 0,519 & $0,945(0,606-1,473)$ & 0,803 \\
\hline Diabetes mellitus & $1,1416(0,986-2,034)$ & 0,060 & $1,163(0,798-1,696)$ & 0,433 \\
\hline Ischemic heart disease & $1,743(1,225-2,480)$ & $<0,01$ & $1,434(0,940-2,189)$ & 0,094 \\
\hline Peripheral vascular disease & $1,602(1,072-2,395)$ & $<0,05$ & $1,484(0,967-2,277)$ & 0,071 \\
\hline Kidney failure & $1,729(0,931-3,211)$ & 0,083 & $0,825(0,419-1,626)$ & 0,579 \\
\hline Heart failure & $2,596(1,749-3,854)$ & $<0,001$ & $1,806(1,052-3,100)$ & $<0,05$ \\
\hline Atrial fibrillation & $1,999(1,375-2,904)$ & $<0,001$ & $1,371(0,873-2,153)$ & 0,171 \\
\hline Symptomatic stenosis & $1,574(1,105-2,240)$ & $<0,05$ & $1,868(1,213-2,876)$ & $<0,01$ \\
\hline
\end{tabular}

RR, Relative risk; CI, Confidence interval. 
All variables in our database were analysed for correlation to outcome. The patients who died from non-cardiovascular causes were

excluded from the regression analysis to avoid competing endpoints. This Cox regression analysis includes all variables with a significant

$p$-value, and age and gender, which were treated as categorical parameters (age: $>75$ years, or $\leq 75$ years). 
Figure 2. Clinical outcome in patients with high grade carotid artery stenosis.

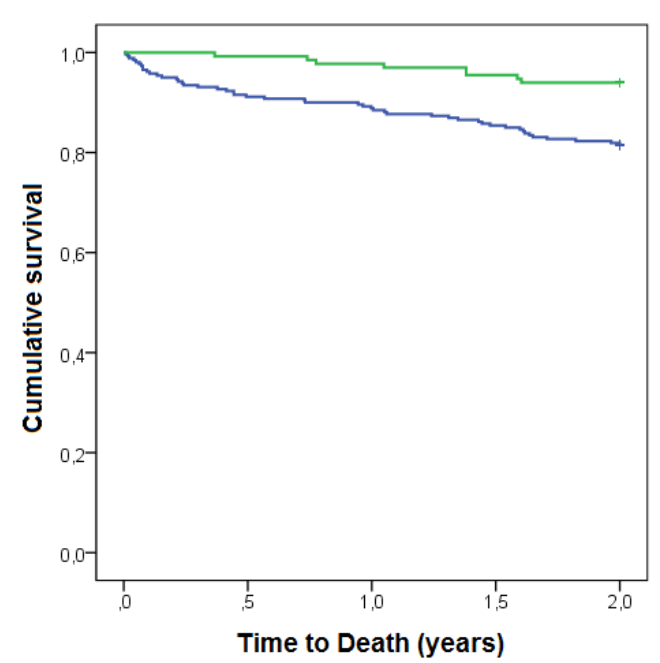

(A)
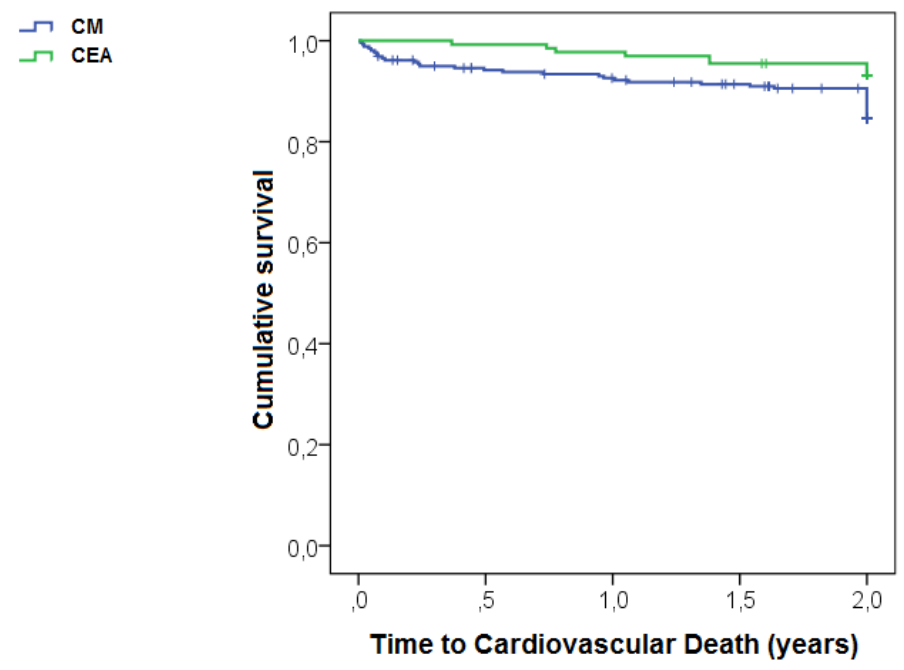

(B)

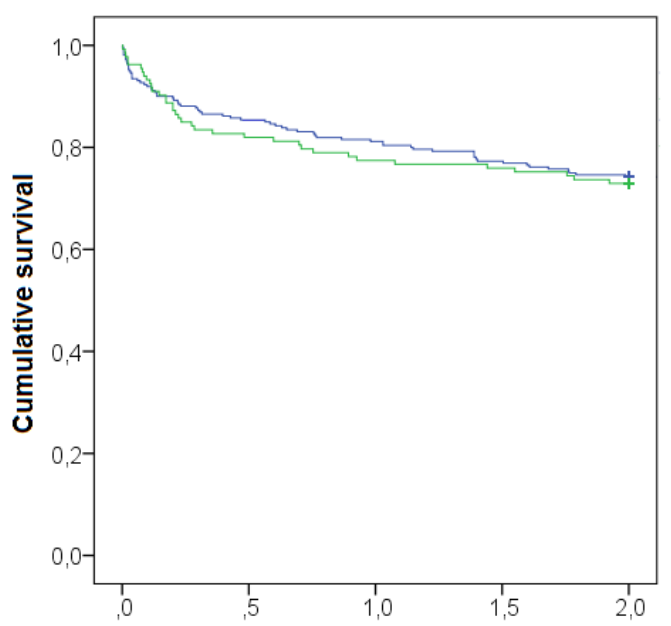

Time to non-fatal Cardiovascular Event (years)

(C)

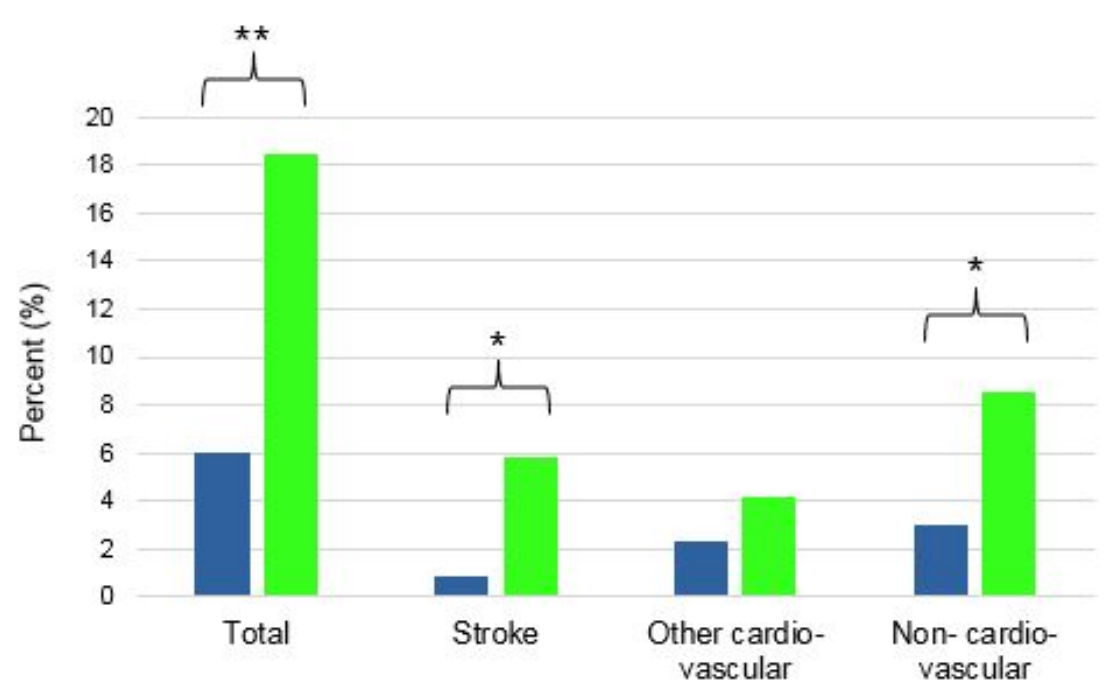

(D)
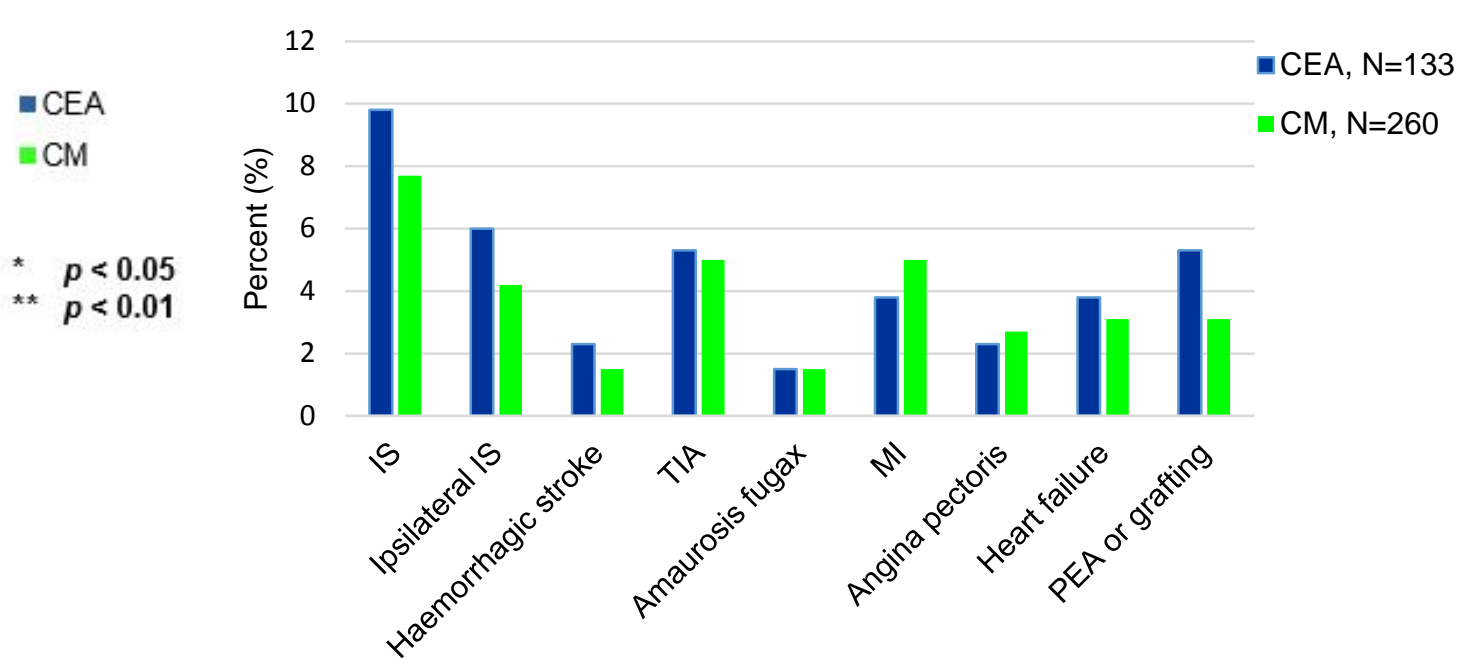

(E)

CEA, Carotid endarterectomy; CM, Conservative management. IS, Ischemic stroke; TIA, Transient ischemic attack; MI, Myocardial infarction; PEA, Peripheral endarterectomy. 


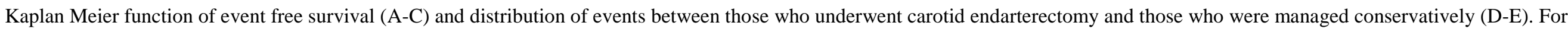

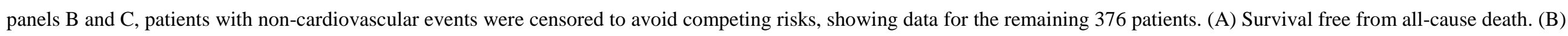

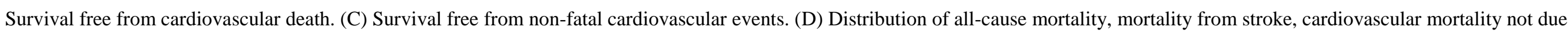
to stroke and non-cardiovascular mortality. (E) Non-fatal cardiovascular events, two-year follow-up ( $p>0.05$ for all comparisons). 
Supplemental Table 1. Definitions

\begin{tabular}{ll}
\hline Variables & Definition \\
\hline
\end{tabular}

Cerebral events

Ischemic stroke

Hemorrhagic stroke

TIA or amaurosis fugax

Cardiac events

Myocardial infarction

New onset angina pectoris
Focal clinical signs of central nervous system dysfunction of vascular origin persisting $\geq 24$ hours

Focal clinical signs of central nervous system dysfunction of vascular origin persisting $\geq 24$ hours with CT and/or MRI scan showing intracerebral hemorrhage

Central nervous system dysfunction or visual impairment lasting $<24$ hours

New diagnosis of STEMI or NSTEMI

a) New diagnosis b) History of chest pain and newly introduced antianginal medication c) History of chest pain and an exercise test positive for cardiac ischemia. Does not apply if the patient was diagnosed with angina and/or already was treated with anti-anginal medication prior to study entry 
New onset heart failure

Lower extremity TEA/or bypass

grafting

New carotid revascularization

Death

Comorbidities

Hypertension

Diabetes

Peripheral events
New diagnosis of congestive heart failure (CHF) or an echocardiogram with evidence of such. This does not apply if the patient was diagnosed with CHF prior to study entry

Vascular surgery including thromb-endarterectomy (TEA), ilio-tibial

bypass, other lower extremity graft or stent implantation

Carotid revascularization (surgery or stent) during follow-up

All deaths, separated into cardiovascular and non-cardiovascular deaths a) Hypertension documented as a diagnosis in the medical record

b) Prescription of anti-hypertensive medication where no heart condition could explain the prescription

a) Diabetes mellitus documented as a diagnosis in the patient's medical record b) Prescription of anti-diabetic medication

CT, Computer tomography; MRI, Magnetic resonance imaging; TIA, Transient ischemic attack; STEMI,

ST-segment elevation myocardial infarction; NSTEMI, Non-ST-segment elevation myocardial infarction; CABG, Coronary artery bypass graft; PCI, Percutaneous coronary intervention; TEA, Thrombendarterectomy. 
For a variable to be registered in the current study, it had to be documented as a diagnosis in the medical record. The only exceptions to this were hypertension and diabetes mellitus, see definitions above. 
Supplemental Table 2. Variables tested for correlation to outcome.

\begin{tabular}{|c|c|}
\hline Variables & Correlation Coefficient $(p)$ \\
\hline Gender & $-0.17(0.742)$ \\
\hline Age & $0.055(0.273)$ \\
\hline Age over 75 & $0.033(0.508)$ \\
\hline Previous cerebrovascular accident & $0.083(0.100)$ \\
\hline Signs of chronic ischemia on computer tomography & $0.065(0.241)$ \\
\hline Symptomatic high-grade carotid stenosis & $0.111(<0.05)$ \\
\hline Endarterectomy & $-0.001(0.990)$ \\
\hline Health care facility where diagnosis was made & $-0.057(0.263)$ \\
\hline Hypertension & $0.064(0.204)$ \\
\hline Systolic blood pressure on target before diagnosis & $0.069(0.213)$ \\
\hline Diastolic blood pressure on target before diagnosis & $0.019(0.736)$ \\
\hline Ischemic heart disease & $0.184(<0.001)$ \\
\hline Periperal artery disease & $0.109(<0.05)$ \\
\hline Diabetes & $0.096(0.057)$ \\
\hline Renal failure & $0.140(<0.01)$ \\
\hline Atrial fibrillation & $0.179(<0.001)$ \\
\hline Obesitas & $0.093(0.124)$ \\
\hline Body mass index & $0.100(0.114)$ \\
\hline Overconsumption of alcohol & $0.019(0.803)$ \\
\hline Smoking & $-0.049(0.364)$ \\
\hline Cancer & $0.052(0.301)$ \\
\hline Metastatic cancer & $0.079(0.117)$ \\
\hline Chronic obstructive pulmonary disease & $0.075(0.136)$ \\
\hline Heart failure & $0.255(<0.001)$ \\
\hline Dementia & $0.003(0.948)$ \\
\hline Connective tissue disease & $-0.050(0.318)$ \\
\hline Previous peptic ulcer & $0.044(0.388)$ \\
\hline
\end{tabular}


These variables were analyzed with a Spearman bivariate correlation test for significant correlation to the combined endpoint of all cardiovascular events and mortality as well as for inter-correlation. Inter-correlation among the significant variables: Symptomatic stenosis is negatively correlated to ischemic heart disease. Ischemic heart disease is positively correlated to peripheral artery disease, renal failure, atrial fibrillation and heart failure. Atrial fibrillation is positively correlated to heart failure. 
Supplemental Table 3. Risk factors at the time of diagnosis of high-grade carotid stenosis.

\begin{tabular}{lccc}
\hline & Patients, N (\%) & Patients, N (\%) & Patients, N (\%) \\
\hline Risk factors at baseline & Yes & No & $\begin{array}{c}\text { Not measured/ } \\
\text { recorded }\end{array}$ \\
\hline & & & \\
TC $\geq 5.0 \mathrm{mmol} / \mathrm{L}$ & $130(33.1)$ & $159(40.5)$ & $104(26.5)$ \\
LDL $\geq 1.8 \mathrm{mmol} / \mathrm{L}$ & $223(56.7)$ & $34(8.7)$ & $136(34.6)$ \\
Current smoking & $100(25.4)$ & $243(61.9)$ & $50(12.7)$ \\
Alcohol overconsumption & $19(4.8)$ & $155(39.2)$ & $219(55.7)$ \\
BMI $\geq 25$ & $156(39.7)$ & $94(23.9)$ & $143(36.4)$ \\
Hypertension & $337(85.8)$ & $56(14.2)$ & - \\
Diabetes mellitus & $126(32.1)$ & $267(67.9)$ & - \\
\hline
\end{tabular}

N, Number of patients; TC, Total cholesterol; LDL, Low-density lipoprotein; BMI, Body mass index.

Risk factors at the time of diagnosis of high grade carotid artery stenosis, based on measurements and documentation in the medical record during the year prior to the diagnosis. 
Supplemental Table 4. Non-cardiovascular mortality

\begin{tabular}{|c|c|c|c|}
\hline & Age & Gender & CEA \\
\hline Respiratory insufficiency & 81 & Male & No \\
\hline Breast cancer & 86 & Female & No \\
\hline Upper GI bleeding due to pancreatic cancer & 63 & Male & No \\
\hline Not specified, forensic autopsy & 68 & Male & Yes \\
\hline Colon cancer and metastatic prostate cancer & 62 & Male & No \\
\hline Pneumonia & 81 & Male & No \\
\hline Generalized malignancy & 81 & Male & No \\
\hline Metastatic penile carcinoma & 65 & Male & No \\
\hline Breast cancer & 69 & Female & No \\
\hline Septic shock & 74 & Male & No \\
\hline Metastatic pancreas cancer & 72 & Male & No \\
\hline Renal failure & 84 & Male & No \\
\hline Cancer in urinary bladder & 70 & Male & No \\
\hline Gangrenous infection & 81 & Female & Yes \\
\hline Not specified & 84 & Male & No \\
\hline Upper GI hemorrhage, circulatory shock & 72 & Male & No \\
\hline Cause of death listed as confidential & 89 & Male & No \\
\hline
\end{tabular}

GI, gastro-intestinal. 
Supplemental Figure 1. Contraindications for surgery in those patients who were managed conservatively.

\section{OTHER CONTRAINDICATIONS}

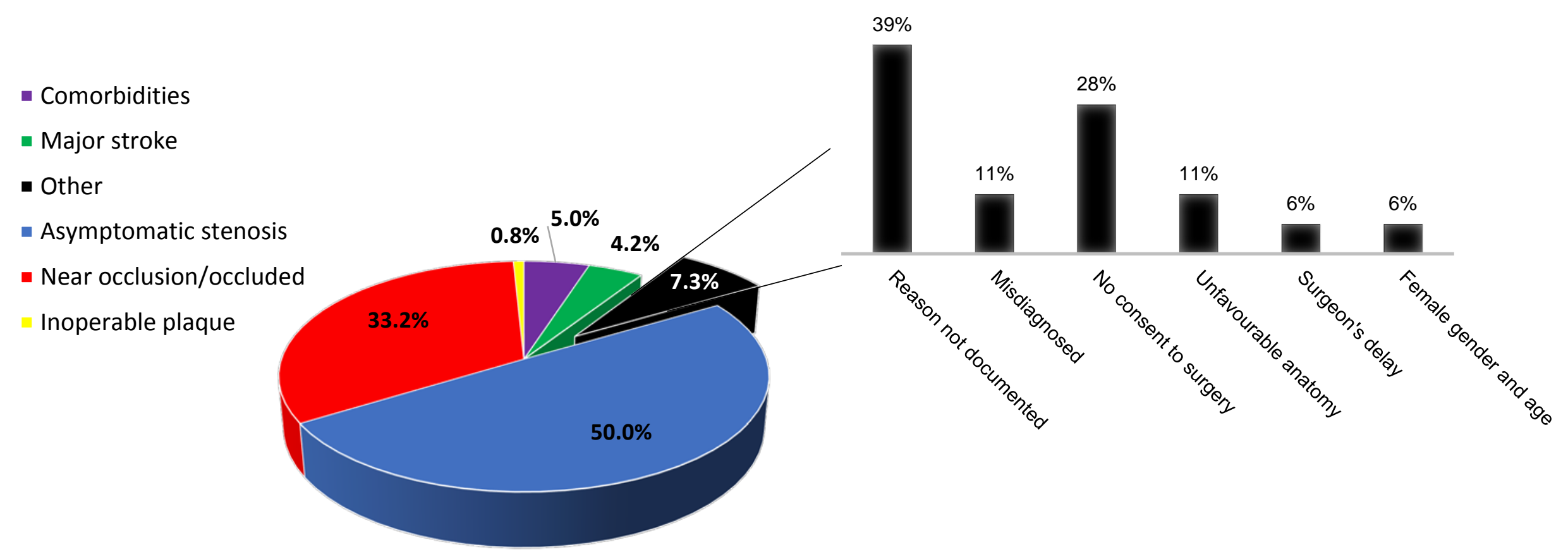

Contraindications for surgery in the conservative treatment group. The pie chart illustrates the distribution of the contraindications. The bar chart specifies the reasons to forego surgery in the category 'other contraindication'. 